\title{
Sistema automático para la clasificación de la opinión pública generada en Twitter
}

\author{
José R. Gálvez-Pérez ${ }^{1}$, Bárbara Gómez-Torreroํㅜ, Raúl I. Ramírez-Chávez ${ }^{1}$, \\ Kathia M. Sánchez-Sandoval ${ }^{1}$, Vicente Castellanos-Cerda ${ }^{1}$, \\ Roberto García-Madrid ${ }^{2}$, and Héctor Jiménez-Salazar ${ }^{1}$ y Esaú Villatoro-Tello ${ }^{1}$ \\ 1 División de Ciencias de la Comunicación y Diseño, \\ Universidad Autónoma Metropolitana Unidad Cuajimalpa, México D.F. \\ 2 División de Ciencias y Artes para el Diseño, \\ Universidad Autónoma Metropolitana Azcapotzalco, México D.F. \\ \{joseramon.galvezperez, barb.torrero, rich1983, kamissonce\}@gmail.com \\ \{vcatellanos, hjimenez, evillatoro\}@correo.cua.uam.mx \\ gmra@correo.azc.uam.mx
}

\begin{abstract}
Resumen. La facilidad de acceso a la diversidad de contenidos hace de Twitter un medio para pronunciarse sobre temas actuales o eventos en tiempo real. Los usuarios de esta red no cumplen únicamente una función pasiva de recepción y consumo de información, sino que al mismo tiempo se convierten en productores de contenidos. El estudio de tuits requiere de una disciplina que permita trabajar con el lenguaje en busca de patrones, los cuales hagan posible proponer una metodología con el fin de analizarlos automáticamente, considerando el contexto en el que han sido publicados. La presente investigación describe un sistema automático para el idioma español que permite conocer la polaridad de la opinión pública manifestada en Twitter respecto a temas políticos de la Ciudad de México.
\end{abstract}

Palabras clave: análisis de opinión, diccionarios afectivos, procesamiento de lenguaje natural, visualización de información, aplicaciones del procesamiento de lenguaje natural.

\section{Introducción}

Hoy en día es indiscutible el papel que están jugando las redes sociales en Internet, que en conjunto con la tecnología, el impacto potencial de la información sobre la vida política, económica y social transforma las dinámicas para comunicarse y adquirir información.

Los medios de comunicación tradicionales ya no son los únicos referentes sobre los acontecimientos del día a día y con el paso del tiempo, se ha incrementado la cantidad de fuentes a las que se tiene acceso para tener mayor conocimiento de lo que sucede en el mundo. Las personas y los usuarios con ayuda de la tecnología, difunden o dan a conocer parte de la información que surge a cada momento. 
En esta línea, Twitter es una red para pronunciarse de forma inmediata sobre temas de cualquier índole que suceden en tiempo real o aquellos que cobran popularidad. Los usuarios de esta red no cumplen únicamente una función de consumo de información, sino que tienen la posibilidad de convertirse en difusores y/o productores de contenidos a través de sus experiencias, testimonios y opiniones personales.

La gran cantidad de información disponible y la velocidad en la que se publica, ha generado dificultades técnicas en el procesamiento para su análisis e interpretación, por lo que se requiere de herramientas que maximicen los beneficios potenciales en esta exploración.

En el presente trabajo, se desarrolló un sistema automático para la recuperación y clasificación de tuits, con el fin de identificar la postura del público de Twitter respecto a temas políticos nacionales. Se describe un sistema automático para el idioma español, que permite acercarse a la polaridad, en forma visual, de la opinión pública de la ciudad de México manifestada en Twitter, teniendo a la mano datos que soporten la toma de decisiones de un usuario especializado en la opinión pública.

El resto de este trabajo está organizado de la siguiente manera: la sección 2 describe brevemente la manera tradicional de atacar el problema de polaridad así como las características de algunos sistemas existentes actualmente. En la sección 3 se describe tanto el recurso léxico como el corpus empleado en el desarrollo de nuestro sistema, de igual forma se describen los resultados obtenidos durante la fase experimental. Posteriormente, en la sección 4 se describen las características del sistema desarrollado así como los resultados de una evaluación realizada con fines de definir el grado de usabilidad de la aplicación propuesta. Finalmente, en la sección 5 se derivan algunas conclusiones e ideas de trabajo futuro.

\section{Antecedentes}

Producto de la gran cantidad de publicaciones que se generan en Twitter, es notorio que el público tiende a bifurcarse entre aquellos que están a favor o en contra de algún acontecimiento. Es por esto que actualmente hay un fuerte interés por parte de especialistas relacionados en las áreas de la mercadotecnia, política, social y de comunicación en contar con herramientas capaces de recuperar mensajes escritos en las redes sociales para su análisis, con el fin de detectar opiniones de los usuarios que permitan mostrar tendencias respecto a determinados temas ${ }^{3}$ Este interés se extiende incluso al público en general que está atento en saber lo que sucede en redes sociales, debido a la inmersión que ha tenido la tecnología en la vida diaria.

En este contexto, existen intentos por desarrollar herramientas que hagan uso de datos obtenidos de Twitter con el fin de identificar la postura de los usuarios respecto a algún tema, los cuales están orientados a trabajar con el idioma inglés en su mayoría. Dentro de los intentos por resolver el problema

\footnotetext{
3 Hasta el año 2014 el foro RepLab representaba el sitio más relevante donde este tipo de sistemas erán evaluados http://nlp.uned.es/replab2014/.
} 
de clasificar opiniones publicadas en Twitter para el idioma español de forma automática, estas empresas se han enfocado a las búsquedas de productos con fines mercadológicos. En este caso, mediante ciertas características sintácticas o semánticas se realiza la clasificación de tuits en positivos, negativos o neutros [1]. También se ha estudiado si los métodos que han sido efectivos para la clasificación de opiniones de tuits en inglés, lo son para español 3. Estos métodos consideran las palabras que se usan en los tuits para darles un valor semántico, las negaciones, e incluso el procesamiento de enlaces a otros sitios web. Sin embargo, este estudio concluyó que los métodos para analizar y clasificar automáticamente tuits en inglés no dieron buenos resultados al implementarlos en tuits escritos, puesto que no se puede establecer un contexto a cada tuit de forma automática.

Como estos trabajos existen muchas propuestas más, las cuales enfocan sus esfuerzos en la búsqueda de formas adecuadas de representación de los documentos así como en la identificación de los atributos que resultan más apropiados en la resolución de la tarea 96].

Con respecto a obtener resultados visuales provenientes del análisis de datos de Twitter, se encuentran disponibles algunas herramientas automáticas en línea que permiten realizar búsquedas de palabras clave presentando resultados cuantitativos sobre la emoción y sentimiento de cada tuit recuperadd 4

Twitter, ha hecho posible enfocarse en elementos particulares que por medio de una visualización geoespacial, muestra seguimiento de palabras o temas en tiempo real en determinada zona geográfica 5 . De esta forma se elige el punto geográfico a nivel mundial sobre el que hay interés en conocer sobre lo que hablan los usuarios.

Estos antecedentes ponen de manifiesto la inquietud por trabajar con información derivada de Twitter, y analizarla con el fin de estudiar las reacciones de la sociedad respecto a algún tema en particular, asimismo, que no hay sistemas para el idioma español que ofrezcan una aproximación a la polaridad de un tópico particular de Twitter. Y aunque pueden ser útiles los métodos empleados en otros sistemas desarrollados para lenguas diferentes al español, orientados a la clasificación de polaridad de tuits, no es viable adaptarlos al español. Por lo anterior, dentro de éste trabajo proponemos y describimos el desarrollo de un sistema que analice la opinión que generan los temas políticos nacionales, basado en un análisis contextual sobre el uso que se le da a Twitter en la Ciudad de México.

\section{Método de clasificación automática de opinión}

Uno de los elementos relevantes para proponer el método de clasificación de opinión fue la conformación de un diccionario afectivo de palabras, donde cada palabra tiene asociado un valor que determina su escala positiva o negativa. Agregado a esto, fue necesario también la construcción de un corpus de trabajo, sobre el cual fuera posible evaluar la pertinencia de método propuesto.

4 http://www.csc.ncsu.edu/faculty/healey/tweet_viz/

5 http://trendsmap.com/topic/\%23cnte 
José R. Gálvez-Pérez, Bárbara Gómez-Torrero, Raúl I. Ramírez-Chávez, et al.

En las siguientes secciones se detalla el proceso de construcción del diccionario como la recolección del corpus de trabajo.

\subsection{Diccionario afectivo}

Estudiar y conocer el contexto fue muy importante para elegir las palabras que integrarían un diccionario que sería la base del funcionamiento del sistema automático propuesto.

Para la conformación del diccionario de palabras que permitiera clasificar los tuits, se inició con la construcción de una lista de palabras obtenida del mismo corpus. Posteriormente se integraron palabras del diccionario Spanish Emotion Lexicon (SEL) proveniente del trabajo "Creación y evaluación de un diccionario marcado con emociones y ponderado para el español" [2]. En dicho trabajo se realizó una investigación a partir del interés de analizar opiniones en las redes sociales con atención a Twitter y presentaron una lista de 2036 palabras en español relacionadas con seis emociones básicas (alegría, sorpresa, repulsión, miedo, enojo, tristeza). Cada palabra de este diccionario tiene asignado un factor de probabilidad de uso afectivo (PFA) que indica el grado en que puede presentar su uso en relación con determinada emoción. Cabe señalar que la escala de valores del PFA es de 0 a 1 , siendo 1 el valor máximo. Así entonces, el diccionario con el cual se trabajó está conformado por una lista de 1443 palabras calificadas con su correspondiente PFA. Es importante mencionar que nuestro diccionario resultó de menor tamaño al de 2] debido a que sólo consideramos las palabras con un PFA con valores entre 0.5 y 1 .

\subsection{Corpus}

En el área de procesamiento de lenguaje natural, existen tareas para las cuales resulta relevante contar con colecciones de datos (textos), los cuales muestran de manera natural ejemplos del uso de la lengua. Este conjunto de documentos llamado "corpus" y a las aplicaciones que utiliza un corpus para obtener las reglas de interpretación, se le conoce como "lingüística basada en corpus".

El corpus de la presente investigación está formado por 2507 tuits recuperados en junio del 2013 y julio del 2014. Los temas recuperados fueron: \#PVEM, Peña Nieto, Hugo Sánchez, CNTE, Reforma Energética, Chapo Guzman y \#EPNvsInternet. Cada tuit fue clasificado de acuerdo a la opinión en consenso por parte de un grupo de cuatro expertos, tomando en cuenta el contexto social y costumbres expresivas de la Ciudad de México. Esto permitió asignar una etiqueta a cada tuit: negativo, positivo o neutro (estos últimos correspondían en su mayoría a las notas informativas de los medios de comunicación en Twitter). El corpus etiquetado fue tomado en cuenta para comparar los resultados obtenidos en los experimentos subsecuentes. Este proceso fue necesario para poder formar un marco de referencia que sirviera para evaluar el desempeño de los métodos propuestos. 
Tabla 1. Muestra de los tuits recuperados y su clasificación asignada por los expertos.

\begin{tabular}{lccccc}
\hline Categoría & \multicolumn{4}{c}{ Entidad y/o tópico principal } \\
\cline { 2 - 6 } de los tuits & Peña_Nieto & Hugo_Sánchez & \#PVEM & CNTE & Total \\
\hline Positivos & 7 & 1 & 7 & 27 & $\mathbf{4 2}$ \\
Negativos & 99 & 71 & 44 & 211 & $\mathbf{4 2 5}$ \\
Neutral & 75 & 29 & 67 & 262 & $\mathbf{4 3 3}$ \\
\hline Total & 181 & 101 & 118 & 500 & $\mathbf{9 0 0}$ \\
\hline
\end{tabular}

\section{3. $\quad$ El método de clasificación}

El método de clasificación de un tuit es determinado tomando en cuenta la ocurrencia de las palabras del diccionario afectivo (Sección 3.1) así como su ponderación afectiva. La polaridad de un tuit se determina por medio de realizar la combinación lineal de los pesos asignados a cada una de las palabras que aparecen dentro de un tuit y que ocurren dentro del diccionario afectivo. La Figura 1 muestra de manera esquemática el algoritmo de clasificación de opiniones.

El método comienza por hacer una comparación entre el tuit en revisión $T$ y una serie de heurísticas que ayudan a determinar cuando un tuit carece de opinión, a las cuales llamamos $T_{I}$ (tuits informativos). En caso de que $T$ contenga características que pertenecen a los $T_{I}$ se le asigna la etiqueta de $T_{W \text { inf }}$ la cual indica que es un tuit informativo, mismo que lo define como un tuit de polaridad neutral. En el caso contrario, si $T$ no tiene características de $T_{I}$, se etiqueta como $T_{W o p}$ indicando ser un tuit de opinión. Es importante mencionar que el conjunto de heurísticas contenidas en $T_{I}$ representa un conjunto de reglas que permiten distinguir cuando un tuit contiene URLs que refieren a sitios formales de información, por ejemplo, periódicos en línea.

Posteriormente se realiza una comparación con de cada palabra contenida en $T$ con el diccionario afectivo $D$ en busca de palabras $\left(D_{w}\right)$ que pertenezcan a $D$. En caso de encontrarlas se identificará el $D_{n u m}$ (valor afectivo de cada palabra) correspondiente de la(s) $D_{w}$, de lo contrario se asignará un valor cero (neutro) a la palabra. En este sentido, un valor afectivo de 0 significa que la palabra en revisión no tiene carga afectiva, i.e., es neutra.

Una vez identificados los $D_{\text {num }}$ en $T$, se realiza una sumatoria para obtener un resultado $N$ (número natural), el cual dependiendo su valor final, indicará la clasificación que tendrá el tuit $T$ en revisión. Si el resultado de la sumatoria es cero, clasificará al tuit como neutro; si es menor a cero se clasificará como negativo y con un valor mayor a cero se le asignará la etiqueta de positivo.

\subsection{Medidas de evaluación}

Para evaluar el método de clasificación propuesto se utilizaron las medidas de precisión $(P)$, recuerdo $(R)$ y medida $\mathrm{F}(F)$, que son medidas comunes en el 


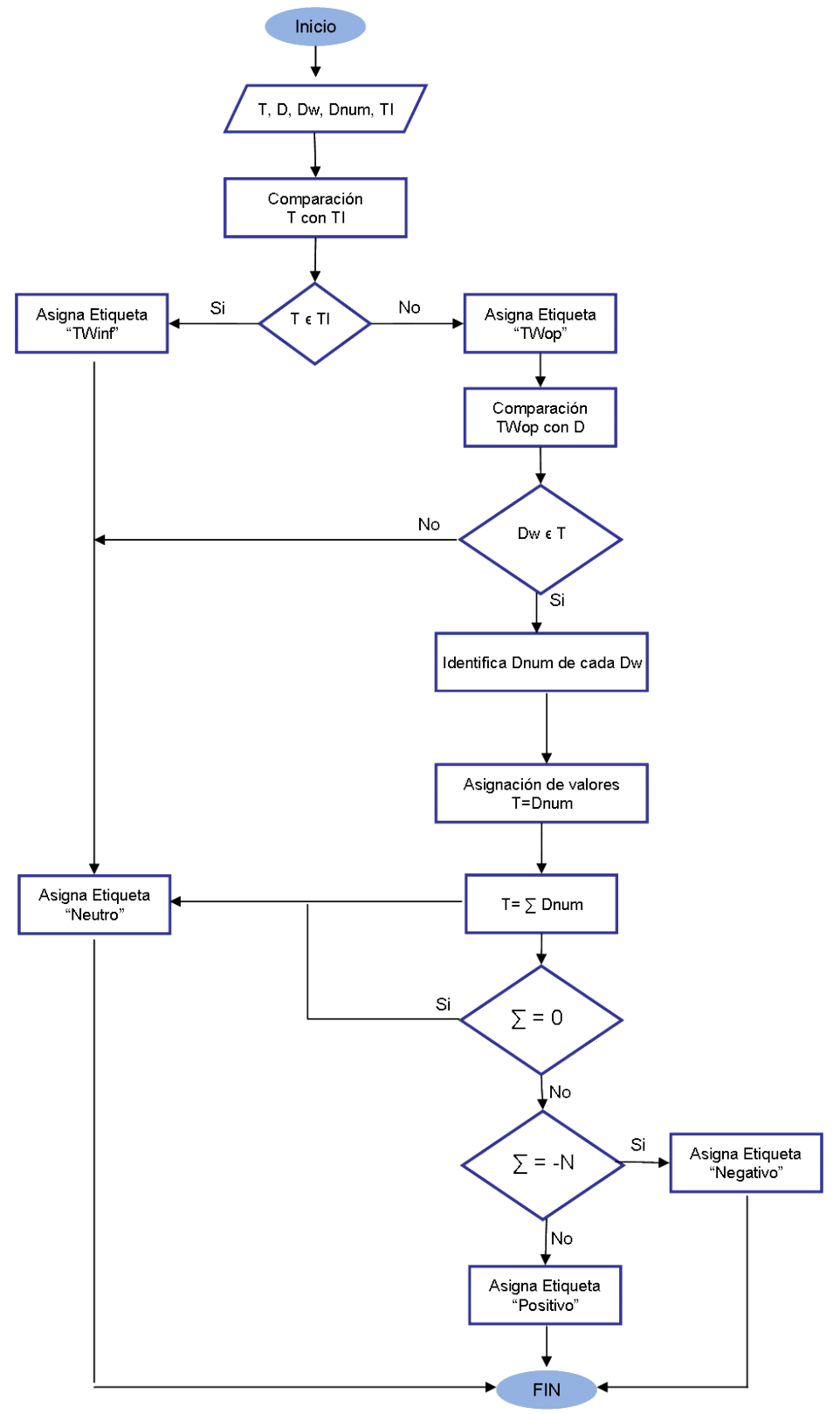

Fig. 1. Diagrama de flujo del algoritmo de clasificación de opinión en Tuiter. Note que de entrada se recibe al tuit $T$, el diccionario $D, D_{w}$ y $D_{n u m}$ refieren a las palabras del diccionario y su correspondiente peso afectivo respectivamente. Finalmente, $T_{I}$ refiere a la ocurrencia de URLs dentro del tuit $T$. 
área de Aprendizaje Automático. La precisión es la proporción de tuits que el sistema clasificó correctamente sobre el total de tuits que deben ser clasificados. El recuerdo es la proporción de los tuits que el sistema clasificó correctamente sobre el total de los tuits que el sistema clasificó [47]. Por último, la exactitud es la proporción del número total de predicciones que son correctas mientras que la medida $\mathrm{F}$ se considera como una medida armónica entre precisión y recuerdo.

La escala de valor que se maneja para cada medida va de 0 a 1 , teniendo como valor máximo 1 . Los resultados obtenidos haciendo uso de estas medidas, son los referentes para comparar el método propuesto y su aplicación en los diferentes experimentos realizados, identificando los casos de aciertos y errores. Para efectos de evaluación de resultados, se ha tomado en cuenta la media $F$ y la Precisión.

\subsection{Evaluación experimental}

Para conocer el desempeño del método de clasificación propuesto, éste se implementó con cada conjunto de tuits recuperado. Teniendo el corpus etiquetado en su totalidad, fue posible comparar y analizar los resultados del método aritmético, con la clasificación de experto con el fin de mejorar el clasificador.

Es importante mencionar que el método propuesto contiene una serie de heurísticas que permiten determinar (con cierta confianza) cuando un tuit es un mensaje informativo y/o objetivo. De esta forma, dichos mensajes no son analizados por el método descrito en la Figura 1, y se evita introducir ruido al sistema de clasificación.

La Tabla 2 muestra los resultados obtenidos de la clasificación de la opinión en el conjunto de tuits descrito en la sección 3.2.

Mediante el proceso de experimentación del clasificador automático, se fueron tomando en cuenta adecuaciones al método propuesto para mejorar su efectividad. La mayor consideración fue en relación al diccionario de términos y su incremento con el fin de tener más elementos que permitan realizar una clasificación de tuits más apropiada.

Las modificaciones que Twitter ha hecho en los últimos años, ponen de manifiesto que trabajar con esta red conlleva ajustes constantes en la metodología de los sistemas de clasificación de opinión. Todo esto se ve reflejado en el uso que se le da a la red, y por consiguiente en la estructura de los tuits, sin embargo, la constante sigue siendo el uso que se le da al lenguaje para manifestar una opinión, lo cual sustenta el trabajo descrito en este artículo.

\section{Sistema automático para la clasificación de la opinión pública}

Una vez que se desarrolló el método para clasificar automáticamente las opiniones emitidas en los mensajes de Twitter, se dio paso al diseño de la parte visual e interactiva del sistema, que incluía la interfaz de uso, así como las distintas visualizaciones presentadas. El objetivo principal era lograr que el 
José R. Gálvez-Pérez, Bárbara Gómez-Torrero, Raúl I. Ramírez-Chávez, et al.

Tabla 2. Evaluación del método de clasificación automática sobre el total de conjuntos recuperados, haciendo uso del método de clasificación definitivo en el cual se considera el total de los tuits recuperados sin separarlos en informativos y de opinión. El \#EPNvsInternet generó mayor opinión por parte de los usuarios y fue con este conjunto donde se obtuvieron los valores más altos en la evaluación.

\begin{tabular}{lccc}
\hline \multirow{2}{*}{ Tópico } & \multicolumn{3}{c}{ Medidas de Evaluación } \\
\cline { 2 - 4 } & $P$ & $R$ & $F$ \\
\hline \#PVEM & 0.568 & 0.590 & 0.458 \\
Hugo_Sánchez & 0.571 & 0.858 & 0.569 \\
Peña_Nieto & 0.658 & 0.634 & 0.641 \\
CNTE & 0.443 & 0.484 & 0.425 \\
Reforma_Energética & 0.558 & 0.547 & 0.521 \\
Chapo_Guzman & 0.581 & 0.553 & 0.557 \\
\#EPNvsInternet & 0.739 & 0.715 & 0.724 \\
\hline
\end{tabular}

usuario conociera la polaridad de opinión respecto a diversas temáticas dentro del contexto político resultante de los mensajes publicados en Twitter. Asimismo, se le presentarían visualizaciones que le permitieran realizar acciones posteriores como el almacenamiento y análisis comparativo de resultados o toma de decisiones con base a ellos.

\subsection{Diseño de la interfaz y las visualizaciones}

La interfaz se refiere a la organización de elementos dispuestos en pantalla mediante los cuales el usuario hará uso del sistema. Su diseño debía enfocarse en lograr que fuera sencilla e intuitiva, sin elementos distractores que facilitaran que la atención del usuario estuviera puesta en los resultados que mostrara el sistema.

Dado lo anterior, la interfaz del sistema se orientó a dirigir al usuario de forma clara y concisa a las opciones que ofrece el sistema para acceder a las distintas gráficas, las cuales proveen distintos maneras de hacer una visualización de la información soportada por el propio sistema 1]. En ellas se despliegan cantidades medibles a través de puntos, líneas, sistema de coordenadas, números, símbolos, palabras y color [10]. Asimismo, las gráficas son presentaciones visuales - breves - que ilustran una o más relaciones entre números, y que nos permiten apreciar relaciones cuantitativas entre muchos elementos y darnos información precisa [5.

Así fue como se realizó una versión digital del prototipo en HTML y PHP para su implementación web. El trabajo en HTML se enfocó a la estructura formal del prototipo, y con PHP se programó el clasificador automático, permitiendo hacer adecuaciones relacionadas con la interfaz y las visualizaciones de información de manera independiente al clasificador. Por otra parte, las visualizaciones son creadas con la librería D3.js, la cual hace posible generar gráficos al momento de obtener los resultados obtenidos de la clasificación automática. La Figura 2 


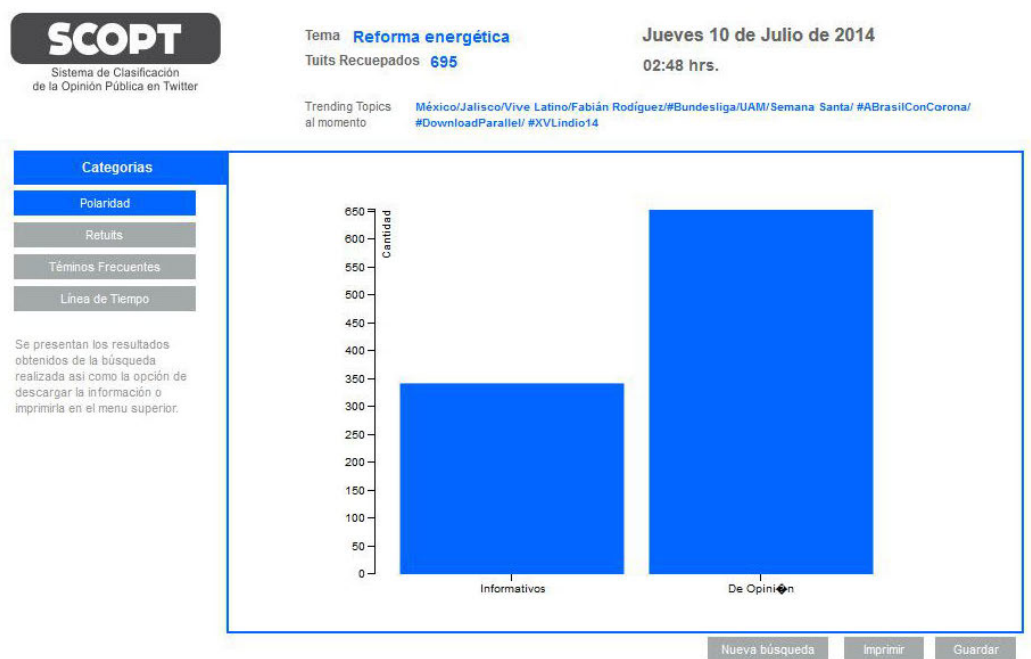

Fig. 2. Pantalla donde se muestran las "Categorías" de los tuits que están siendo analizados. Las categorias corresponden a tuits informativos o tuits de opinión. El sistema SCOPT sólo determina la polaridad de aquellos tuits que se consideran subjetivos, i.e., tuits de opinión.

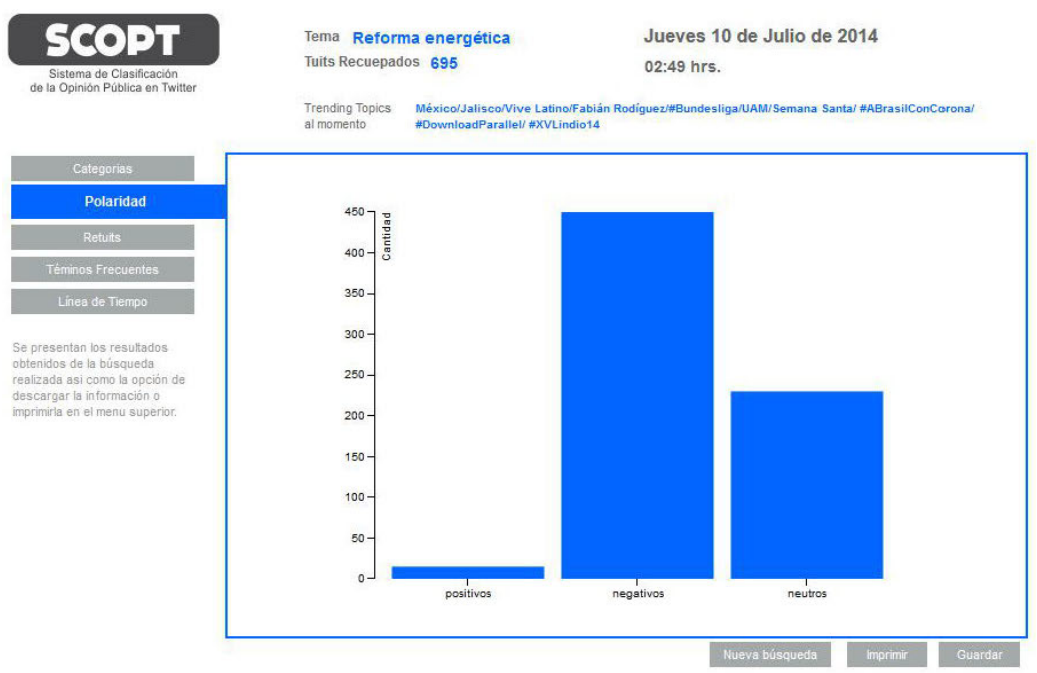

Fig. 3. Ejemplo de visualización "Polaridad". Aquí el usuario puede conocer rápidamente la cantidad de tuits positivos, negativos y/o neutrales existentes en la muestra de tuits que están siendo analizados. 
muestra unos ejemplos de las pantallas principales del sistema SCOPT, mismo que actualmente está alojado en http://lyr.cua.uam.mx

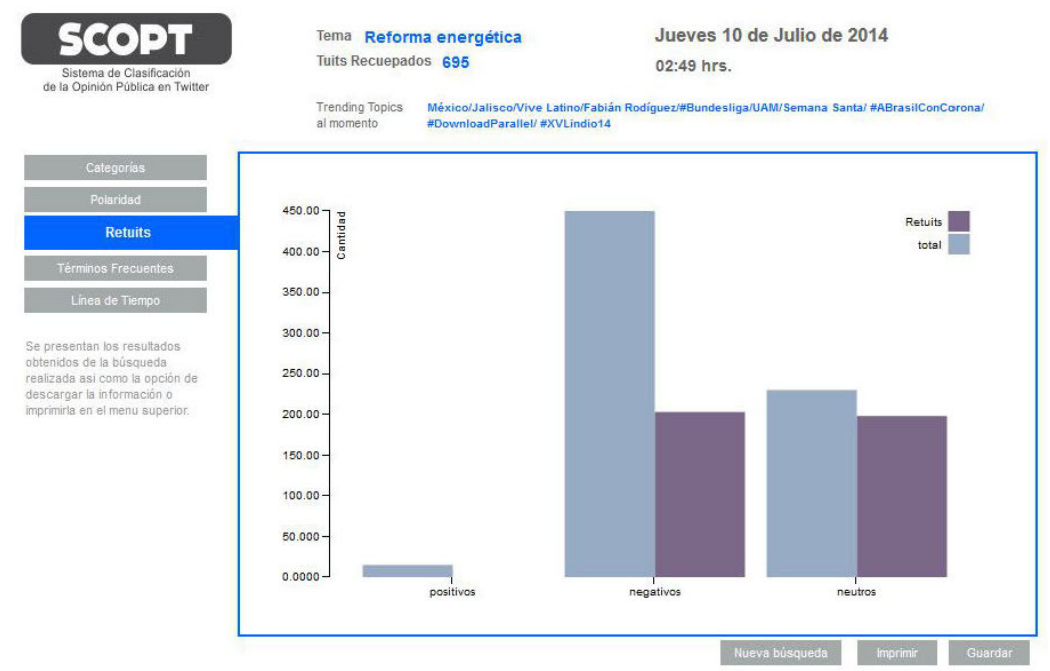

Fig. 4. Ejemplo de visualización "Retuits". En esta pantalla el usuario puede valorar la polaridad de los tuits que están siendo analizados conociendo cuántos de éstos son resultado de un retuit.

\subsection{Comprobación y refinamiento}

La evaluación tiene el propósito de conocer problemas de usabilidad y aplicar un proceso iterativo de ajustes al prototipo antes de la implementación del sistema. En esta etapa la propuesta se puso a prueba con usuarios potenciales con el fin de recabar información valiosa para su mejoramiento. La serie de ajustes y refinamientos se siguieron hasta lograr un nivel óptimo de eficiencia.

Las pruebas de "eye tracking" pueden resultar valiosas para constatar adónde se dirige la atención de las personas que están haciendo uso del sistema. "El concepto de eye-tracking hace referencia a un conjunto de tecnologías que permiten monitorizar y registrar la forma en que una persona mira una determinada escena o imagen, en concreto en qué áreas fija su atención, durante cuánto tiempo y qué orden sigue en su exploración visual" 8. El eye-tracking es una tecnología de seguimiento ocular con mucho auge en el mundo de la usabilidad. Si bien los datos obtenidos mediante estas pruebas nos permiten saber dónde fija su atención el usuario, y qué zonas pasan desapercibidas, esta información puede resultar limitada porque no explica las causas por las que esto pasa, así que conviene establecer algún vínculo entre fijaciones y actividad cognitiva. Por ello, se pensó combinar la aplicación de un test de tareas de forma complementaria 


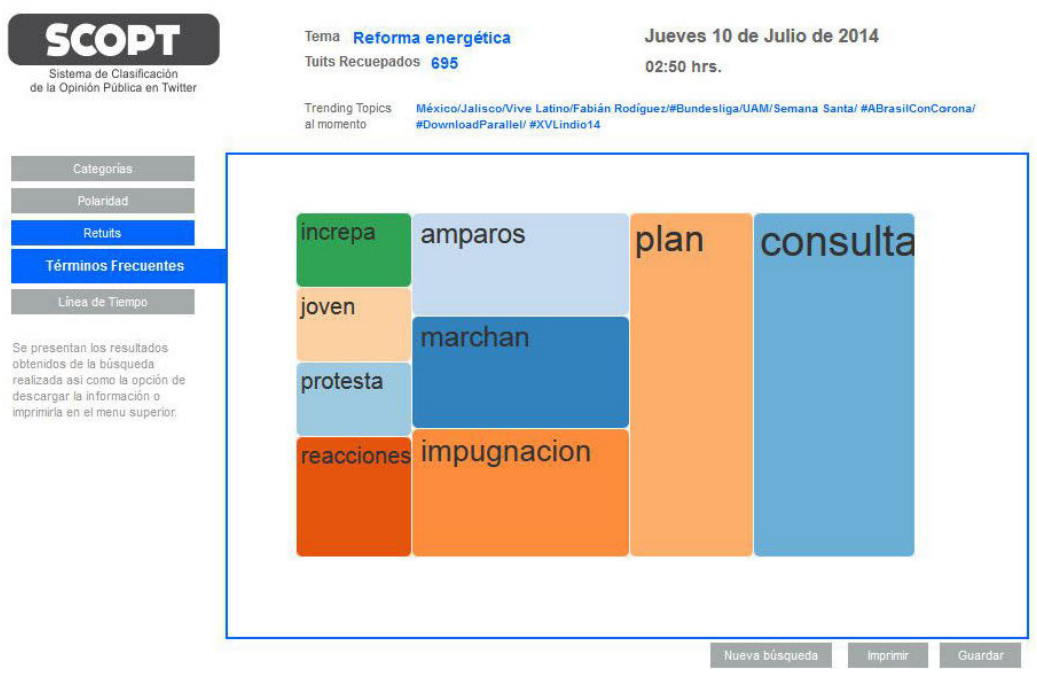

Fig. 5. Ejemplo de visualización de los "Términos frecuentes". El objetivo de esta pantalla es proporcionar al usuario un vistazo rápido de los términos más comúnmente utilizados en la muestra de tuits en revisión. Idealmente darán una idea intuitiva de la temática de los mensajes.

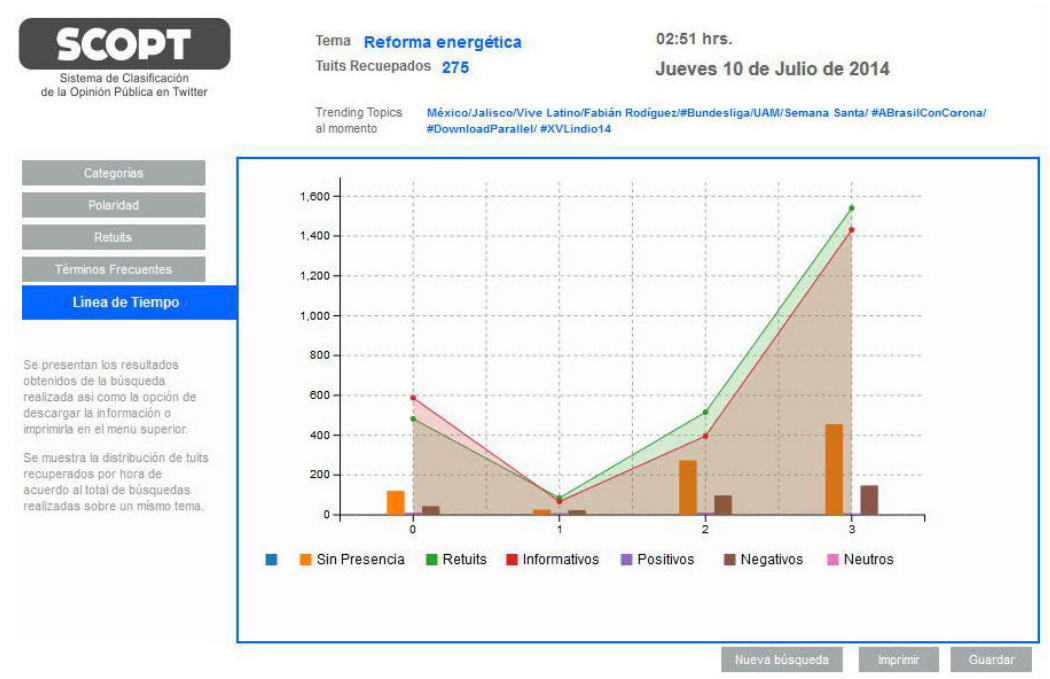

Fig. 6. Ejemplo de visualización de la "Línea de Tiempo". En esta pantalla el usuario puede consultar el historial de sus búsquedas realizadas y comparar gráficamente los cambios de polaridad que han sucedido desde la primera búsqueda. 
con la prueba de eye-tracking, pues cada uno aporta información exclusiva y facilitaría la interpretación de los datos obtenidos. En la Figura 3 se muestran las distintas pantallas que se presentan al usuario, con representaciones visuales de los recorridos de los cuatro participantes evaluados.

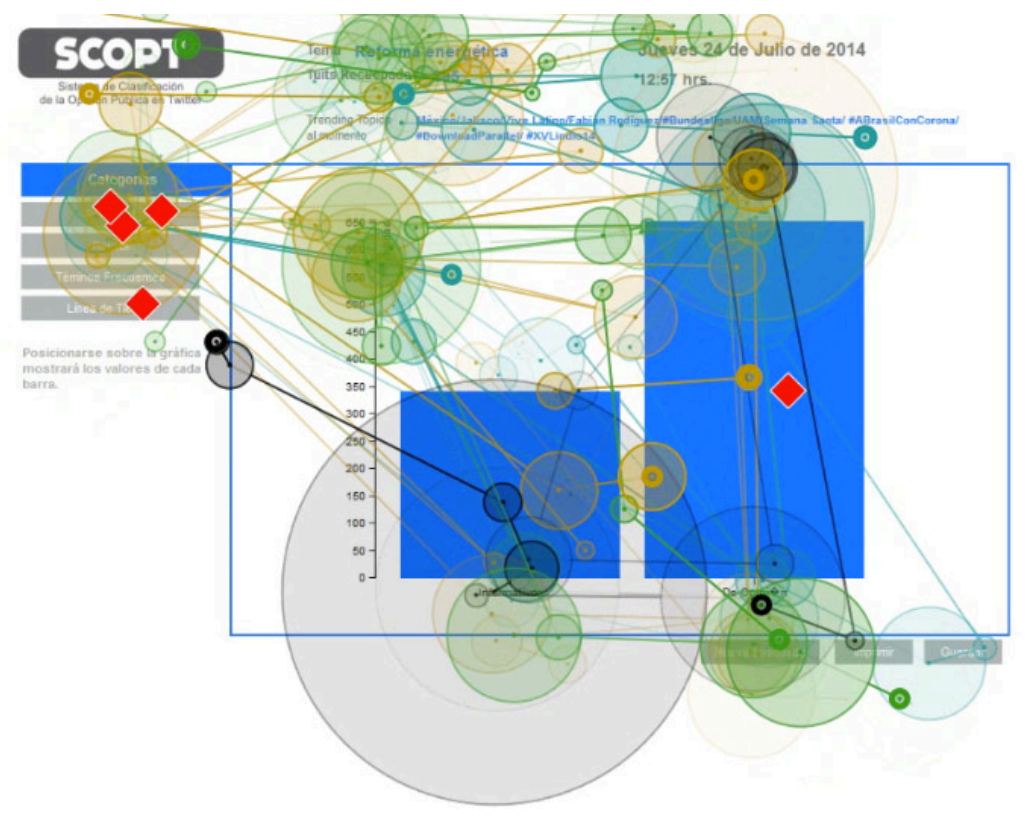

Fig. 7. Recorridos visuales de los participantes evaluados en la pantalla de "Categorías de Tuits (Informativo VS. Opinión)". Evaluación registrada por medio del sistema de eye-tracking.

Entre los resultados que arrojó esta etapa de evaluación dentro del proceso de diseño de la herramienta, encontramos que la interfaz cuenta con un diseño muy sintético y sin mayor problema para localizar, leer y entender la información mostrada, pues no se presentan elementos distractores dentro de la pantalla que desvíen la atención del usuario.

\section{Conclusiones y trabajo futuro}

En este trabajo, dentro de los temas políticos en la Ciudad de México, se pudo constatar que Twitter es un canal de opinión: sus usuarios a partir de las publicaciones que emite y hace lectura, estimula la continuidad de la discusión de un tema, nutre sus comentarios o refuerza su postura con la incorporación de material multimedia y enlaces web.

La exploración de los tuits generados ante eventos políticos nacionales, permitió descubrir posibles patrones en las formas de opinar y dependiendo del 


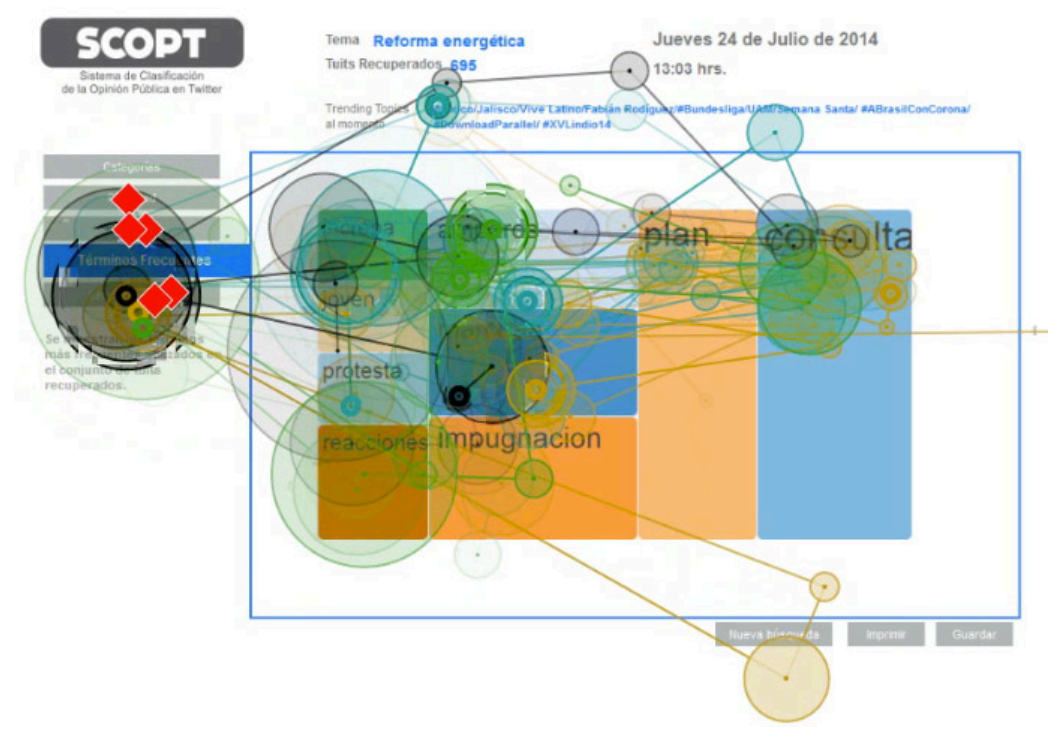

Fig. 8. Recorridos visuales de los participantes evaluados en la pantalla de "Términos Frecuentes". Evaluación registrada por medio del sistema de eye-tracking.

contexto y el tema se obtuvieron palabras que permitirían encontrar la polaridad de un tuit, permitiendo así la conformación del diccionario afectivo, mismo que es la base del sistema de clasificación propuesto. Entre mayor era la cantidad de palabras que integraban el diccionario de términos, mayor fue la precisión del mismo. Esto se hizo notorio conforme se fueron realizando los experimentos, ya que en cada uno de ellos, el diccionario iba incrementándose. Esta relación reforzó el método propuesto y propició continuar la búsqueda de palabras que hicieran posible determinar la polaridad de un tuit.

En este orden de ideas, para el diseño de interfaz del nuestro sistema, se retomaron los resultados arrojados mediante el cuestionario aplicado y la prueba de eye-tracking, permitiendo mostrar visualizaciones claras, limpias y muy sintéticas, por tanto una correcta lectura y comprensión de la información presentada. Si bien, aún podría pulirse la propuesta y aumentar la interactividad en las gráficas, el sistema arroja información general comprensible para los usuarios especializados, y sin que esto excluya al usuario general.

La generación de un método eficiente, derivado de los estudios y análisis previos, nos permitieron desarrollar SCOPT, bajo el propósito de tener un sistema sencillo de usar, presentando resultados de forma visual además de contar con la posibilidad de descargar la información obtenida para analizarla más puntualmente en caso de que así se requiera. Es importante mencionar que éste tipo de herramientas se vuelven fundamentales para el experto en análisis de la opinión publica, pues le permite de manera rápida y sencilla orientar su trabajo de investigación hacia aquellos temas que son de su interés al mismo tiempo 
que le proporciona una aproximación sobre el sentir de la población hacia dicho tema.

Como trabajo futuro planeamos incorporar a la aplicación SCOPT técnicas más sofisticadas de clasificación de polaridad, como lo podría ser la inclusión de atributos estilísticos. De igual forma nos interesa incrementar las heurísticas que ayudan a determinar cuando un tuit es de opinión o informativo. Es conveniente mencionar que el problema de ironía aún no es soportado por el sistema desarrollado, consideramos que el desarrollo de más recursos lingüísticos en combinación con formas alternativas de representación podría ayudar a atacar éste problema.

Agradecimientos. El presente trabajo fue realizado con el apoyo de CONACyT (Becas: 373288, 373287, 373284, 373285). Agradecemos también al programa de Maestría en Diseño, Información y Comunicación (MADIC) de la Universidad Autónoma Metropolitana Unidad Cuajimalpa, así como al SNI-CONACyT.

\section{Referencias}

1. Card, S.K., Mackinlay, J.D., Shneiderman, B. (eds.): Readings in Information Visualization: Using Vision to Think. Morgan Kaufmann Publishers Inc., San Francisco, CA, USA (1999)

2. Díaz-Rangel, I., Sidorov, G., Suárez-Guerra, S.: Creación y evaluación de un diccionario marcado con emociones y ponderado para el español (creation and evaluation of a dictionary tagged with emotions and weighted for spanish). Onomázein, Revista de Lingüística, Filología y Traducción (29), 1-26 (2014)

3. Fernández, A., Nuñez, L., Morere, P., Santos, A.: Sentiment analysis and topic detection of spanish tweets: A comparative study of nlp techniques. Revista de Procesamiento del Lenguaje Natural (50), 45-52 (2013)

4. Hernández, J., Ramírez, J., Ferri, C.: Introducción a la minería de datos. Prentice Hall, Pearson Educación, S.A. (2006)

5. Kosslyn, S.: Graph Design for the Eye and the Mind. Oxford University Press (2006)

6. Leon-Martagón, G., Villatoro-Tello, E., Jiménez-Salazar, H., Sánchez-Sánchez, C.: Análisis de polaridad en twitter. Research in Computing Science 62, 69-78 (2013)

7. Lewis, D.: Evaluating text categorization. In: Proceedings of Speech and Natural Language Workshop. pp. 312-318 (1991)

8. Page, W.: No solo usabilidad: Revista sobre personas, diseño y tecnología, uRL: http://www.nosolousabilidad.com/articulos/eyetracking.htm\#sthash.7N1RrSks.dpuf

9. Sidorov, G., Miranda-Jiménez, S., Viveros-Jiménez, F., Gelbukh, A., CastroSánchez, N., Velásquez, F., Díaz-Rangel, I.and Suárez-Guerra, S., Treviño, A., Gordon, J.: Empirical study of machine learning based approach for opinion mining in tweets. In: Lecture Notes in Artificial Intelligence LNAI. vol. 7629, pp. 1-14 (2012)

10. Tufte, E.: The Graphic Display of Quantitative Information. Graphics press (1983)

11. Valderrábanos, A., Torrejón, E.: Natural opinions: extracción de opinión basada en pln para contenidos generados por usuarios. In: CEUR Workshop Proceedings. pp. 339-346. No. 697 (2010) 